\title{
Article \\ Effects of Aspergillus fumigatus Conidia on Apoptosis and Proliferation in an In Vitro Model of the Lung Microenvironment
}

\author{
Hisako Kushima ${ }^{1,2, *,+}$, Toshiyuki Tsunoda ${ }^{3,4,+}$, Taichi Matsumoto ${ }^{5}$, Yoshiaki Kinoshita ${ }^{2} \mathbb{D}$, Koichi Izumikawa ${ }^{6}$, \\ Senji Shirasawa ${ }^{3,4} \mathbb{D}$, Masaki Fujita $^{1}$ and Hiroshi Ishii ${ }^{2}$ (D) \\ check for \\ updates \\ 1 Department of Respiratory Medicine, Faculty of Medicine, Fukuoka University, Fukuoka 814-0180, Japan; \\ mfujita@fukuoka-u.ac.jp \\ 2 Department of Respiratory Medicine, Fukuoka University Chikushi Hospital, Fukuoka 818-8502, Japan; \\ y3kinoshita@fukuoka-u.ac.jp (Y.K.); hishii@fukuoka-u.ac.jp (H.I.) \\ 3 Department of Cell Biology, Faculty of Medicine, Fukuoka University, Fukuoka 814-0180, Japan; \\ tsunoda@fukuoka-u.ac.jp (T.T.); sshirasa@fukuoka-u.ac.jp (S.S.) \\ 4 Department of Central Research Institute for Advanced Molecular Medicine, Fukuoka University, \\ Fukuoka 814-0180, Japan \\ 5 Department of Pharmaceutical Sciences, Faculty of Drug Informatics and Translational Research, Fukuoka \\ University, Fukuoka 814-0180, Japan; tmatsumoto@fukuoka-u.ac.jp \\ 6 Department of Infectious Diseases, Nagasaki University Graduate School of Biomedical Sciences, \\ Nagasaki 852-8501, Japan; koizumik@nagasaki-u.ac.jp \\ * Correspondence: hkushi@fukuoka-u.ac.jp; Tel.: +81-92-921-1011; Fax: +81-92-928-3890 \\ + These authors contributed equally to the research.
}

Citation: Kushima, H.; Tsunoda, T.; Matsumoto, T.; Kinoshita, Y.; Izumikawa, K.; Shirasawa, S.; Fujita, M.; Ishii, H. Effects of Aspergillus fumigatus Conidia on Apoptosis and Proliferation in an In Vitro Model of the Lung Microenvironment. Microorganisms 2021, 9, 1435. https://doi.org/ $10.3390 /$ microorganisms 9071435

Academic Editor: Eric Dannaoui

Received: 31 May 2021

Accepted: 1 July 2021

Published: 2 July 2021

Publisher's Note: MDPI stays neutral with regard to jurisdictional claims in published maps and institutional affiliations.

Copyright: (c) 2021 by the authors. Licensee MDPI, Basel, Switzerland. This article is an open access article distributed under the terms and conditions of the Creative Commons Attribution (CC BY) license (https:// creativecommons.org/licenses/by/ $4.0 /)$.

\begin{abstract}
Background/Aim: Aspergillus is often detected in respiratory samples from patients with chronic respiratory diseases, including pulmonary fibrosis, suggesting that it can easily colonize the airways. To determine the role of Aspergillus colonization in pulmonary fibrosis, we cultured human lung epithelial A549 cells or murine embryo fibroblast NIH/3T3 cells with Aspergillus conidia in 3D floating culture representing the microenvironment. Materials and Methods: Cells were cultured in two-dimensional (2D) and three-dimensional floating (3DF) culture with heat-inactivated Aspergillus fumigatus (AF) 293 conidia at an effector-to-target cell ratio of 1:10 (early-phase model) and 1:100 (colonization model), and RNA-sequencing and Western blots (WB) were performed. Results: AF293 conidia reduced A549 cell growth in 2D and 3DF cultures and induced apoptosis in A549 spheroids in 3DF culture. RNA-sequencing revealed the increased expression of genes associated with interferon-mediated antiviral responses including MX dymamin-like GTPase 1 (MX1). Interestingly, the decreased expression of genes associated with the cell cycle was observed with a high concentration of AF293 conidia. WB revealed that epithelial-mesenchymal transition was not involved. Notably, AF293 conidia increased NIH/3T3 growth only in 3DF culture without inducing an apoptotic reaction. RNA-sequencing revealed the increased expression of genes associated with interferon signalling, including MX2; however, the decreased expression of genes associated with the cell cycle was not observed. Conclusions: AF affects both apoptosis of epithelial cells and the growth of fibroblasts. A deeper understanding of the detailed mechanisms underlying Aspergillusmediated signaling pathway in epithelial cells and fibroblasts will help us to understand the lung microenvironment.
\end{abstract}

Keywords: Aspergillus fumigatus; lung epithelial cells; fibroblast; apoptosis; proliferation

\section{Introduction}

Aspergillus species (spp.) are universally and widely present in the environment, including air-conditioning units, compost, and damp or flood-damaged housing and hospital building projects [1]. Although invasive pulmonary aspergillosis is a fatal disease caused 
by Aspergillus spp. in immunocompromised hosts, airway colonization by Aspergillus spp. is observed in approximately half of patients in an adult pneumology ward despite no typical symptoms of aspergillosis [2,3]. Among cases of chronic respiratory disease, such as intestinal pneumonia/pulmonary fibrosis, $71 \%$ of patients did not develop any kind of aspergillosis despite Aspergillus colonization in the airway [2,3], suggesting an unknown association between Aspergillus colonization and the development and progression of lung fibrosis other than aspergillosis.

Idiopathic pulmonary fibrosis is well known to be a refractory and progressive lung disease with a poor prognosis $[4,5]$. Several molecular mechanisms have been hypothesized to underlie the development of pulmonary fibrosis, such as apoptosis and/or epithelialmesenchymal transition (EMT) of pulmonary epithelial cells; however, most upstream triggers of the disease remain unclear [6-11].

Among Aspergillus spp., Aspergillus fumigatus (AF) is reported to be more evident in pulmonary fibrosis [12]. A previous in vitro study addressed the association between AF and pulmonary fibrosis using AF conidia and lung epithelial cells; however, the AF conidia used these studies were heat inactivated at high temperature $\left(90,120^{\circ} \mathrm{C}\right)$ or killed $[13,14]$. Furthermore, these studies were only performed in a two-dimensional (2D) culture. In the present study, to examine the interaction between AF conidia and epithelial cells or fibroblasts in a pathophysiological state, we treated AF conidia at low temperature $\left(70^{\circ} \mathrm{C}\right)$ and applied both 2D and three-dimensional (3D) floating (3DF) co-culture methods, which seem to resemble the lung microenvironment [15], at an effector-to-target cell ratio of 1:10 (early-phase model) and 1:100 (colonization model).

\section{Materials and Methods}

\subsection{Antibodies}

An EMT antibody sampler kit, including anti-ZO-1 antibody, anti-vimentin antibody and anti-e-cadherin, was purchased from Cell Signaling (Tokyo, Japan). Anti-actin antibody was purchased from Sigma-Aldrich (St. Louis, MO, USA).

\subsection{Suspension of AF293 Conidia}

A. fumigatus (AF293), a widely used reference strain, was kindly provided by the Department of Infectious Diseases, Nagasaki University Graduate School of Biomedical Sciences, Nagasaki, Japan. The strain was isolated from the lung of a leukopenic patient who died with invasive pulmonary aspergillosis at the university. For experiments, cultures of AF293 isolate were maintained in potato dextrose agar. After five days at room temperature, the cells were harvested. The harvested cells were suspended in $5 \mathrm{~mL}$ of phosphate-buffered saline (PBS) with $1 \%$ Tween 20 and then filtered through a cell strainer. In this process, the agar and hyphae were removed, and only the conidia were collected. The conidia were precipitated by centrifugation at $3500 \mathrm{rpm}$ for $5 \mathrm{~min}$, and then the supernatant was discarded. After washing twice in $5 \mathrm{~mL}$ of PBS with $1 \%$ Tween 20, the sample was suspended in an appropriate amount of PBS (about $4 \mathrm{~mL}$ ) and adjusted to $10^{8}$ cells per milliliter of PBS. The conidia were then inactivated by heating at $70^{\circ} \mathrm{C}$ for $1 \mathrm{~h}$. Killing was determined by plating on agar.

\subsection{A Two-Dimensional (2D) Proliferation Assay}

A549 cells (human alveolar adenocarcinoma cell line) as a model of type II pneumocytes and mouse NIH/3T3 fibroblasts (200,000 cells) were seeded in $10 \mathrm{~cm}$ dishes (Corning Inc., Corning, NY, USA) [16]. Cells were cultured at $37{ }^{\circ} \mathrm{C}$ in $5 \% \mathrm{CO}_{2}$ with or without heat-inactivated AF293 conidia at an effector-to-target cell (E:T) ratio of 1:10 and 1:100, as described previously [17]. Images of cells were taken using a BZ-X700 (Keyence, Osaka, Japan) on days 0 and 3 . The cells were also trypsin-treated and trypan blue-stained on day 3 , and the number of cells was counted. 


\subsection{Three-Dimensional Floating (3DF) Cell Culture}

A549 and mouse NIH/3T3 cells (2000 cells/well) were seeded in round-bottomed 96-well plates with ultra-low-attachment surfaces (Corning Inc.). Cells were cultured in a $\mathrm{CO}_{2}$ incubator with or without heat-inactivated AF293 conidia at an E:T ratio of 1:10 and 1:100, as described previously [15,17-19]. Images of cells were taken using a BZX700 (Keyence), and the spheroid area was measured using a BZ Analyzer (Keyence), as described previously $[15,20]$. The growth rates of tumor spheroids were calculated based on the changes in the spheroid area on days 3 and 7.

\subsection{Detection of Apoptotic Cells in Spheroids}

Apoptotic cells were stained on day 7 using a CellEvent ${ }^{\mathrm{TM}}$ Caspase-3/7 Green Detection Kit (Invitrogen, Thermo Fisher Scientific, Waltham, MA, USA) according to the manufacturer's instructions. Cells were imaged using a BZ-X700 (Keyence). Integration of the fluorescence intensity/area were measured using a BZ-X700 Analyzer (Keyence).

\subsection{Western Blotting}

Cells were lysed in Radioimmnunoprecipitation assay buffer (RIPA) buffer (50 mM Tris- $\mathrm{HCl}, \mathrm{pH} 7.5,150 \mathrm{mM} \mathrm{NaCl}, 1 \% \mathrm{NP} 40,0.5 \%$ sodium deoxycholate, $0.1 \%$ sodium dodecyl sulfate, and protease inhibitor cocktail [Roche, Basel, Switzerland]) and subjected to immunoblotting, as described previously [20]. Quantitative analyses of the immunoblots were performed using the ImageJ 1.52a software program (National Institutes of Health, Bethesda, MD, USA).

\subsection{RNA Sequencing Analysis (RNA-Seq)}

RNA-seq was performed by Kyushu Prosearch LLP (Fukuoka, Japan). In brief, total RNA was extracted from A549 cells and NIH/3T3 cells at day 3 using TRIzol reagent (Invitrogen, Thermo Fisher Scientific) according to the manufacturer's instruction. The RNA quality was examined using an RNA 6000 Pico kit (Agilent, Santa Clara, CA, USA). An amount of $500 \mathrm{ng}$ total RNA was used for RNA-seq library preparation with NEBNext rRNA Depletion Kit and NEBNext Ultra Directional RNA Library Prep Kit (New England Biolabs, Ipswich, MA, USA). Paired-end sequencing $(2 \times 36$ bases $)$ was performed with a NextSeq500 (Illumina, San Diego, CA, USA). Reads were mapped on hg19 human reference genome and quantified using CLC Genomics Workbench v10.1.1 (Qiagen, Redwood City, CA, USA).

\subsection{Real-Time Quantitative Reverse Transcription-Polymerase Chain Reaction ( $q R T-P C R$ )}

Total RNA was extracted using TRIzol (Thermo Fisher Scientific K.K., Tokyo, Japan) before synthesis of cDNA using SuperScript IV VILO Master Mix (Thermo Fisher Scientific K.K.). cDNA was amplified using THUNDERBIRD ${ }^{\circledR}$ SYBR qPCR Mix (TOYOBO CO., LTD., Osaka, Japan) and gene-specific primers, as described previously [20]. The gene amplification was monitored by quantitative polymerase chain reaction (PCR) was performed using Step One Plus real-time PCR System (Thermo Fisher Scientific K.K.). The PCR primers used for each of the genes were as follow: MX1-Sense, $5^{\prime}$-GGCTGTTTACCAGACTCCGACA- $3^{\prime}$ and MX1-Antisense, $5^{\prime}$-CAC AAAGCCTGGCAGCTCTCTA- $3^{\prime}$; OASL-Sense, $5^{\prime}$-CACCATTG TGCCTGCCTACA- ${ }^{\prime}$ and OASL-Antisense, $5^{\prime}$-TCACGAAATTTCTCTGCAGC-3'; TRIM22Sense, $5^{\prime}$-GGATCGTCAGTAGAGATGCTGC- ${ }^{\prime}$ and TRIM22-Antisense, $5^{\prime}$-GAACTTGCAG CATCCCACTCAG-3'; RSAD2-Sense, 5' -AGG AAGGTGAGGTGAATGTG-3' and RSAD2Antisense, 5' -TGGCTTCTTCAATGTCCAGC-3'; GAPDH-Sense, 5' -TCTGCTCCTCCTGTTC GAACA-3' and GAPDH-Antisense, 5'-AAAAGCAGCCCTGGTGACC-3'.

\subsection{Ontology Analyses}

Ontology analyses were performed using ConsensusPathDB-human according to interactions [21]. In brief, a gene set analysis was performed by an over-representation 
analysis. The gene identifier was set to the gene symbol, and pathways were defined by the Reactome Pathway Database.

\subsection{Statistical Analyses in Cell Culture Experiments}

Statistical analyses were performed using unpaired two-tailed Student's $t$-tests. All $p$-values less than 0.05 were considered statistically significant.

\section{Results}

\subsection{AF293 Conidia Suppress the Growth of A549 Cells}

The control A549 cells became monolayered and adherent as the days passed. However, no such morphological changes occurred in the A549 with AF293 heat-inactivated conidia (Figure 1A). The numbers of AF293 conidia used for this experiment were 10-fold (1:10) and 100-fold (1:100) higher than those of A549 cells. In 2D culture, the relative numbers of cells with AF293 conidia at ratios of 1:10 and 1:100 were 0.407- and 0.176-fold smaller than those of the control, respectively (Figure 1A; ${ }^{* *} p<0.001$ ).

A
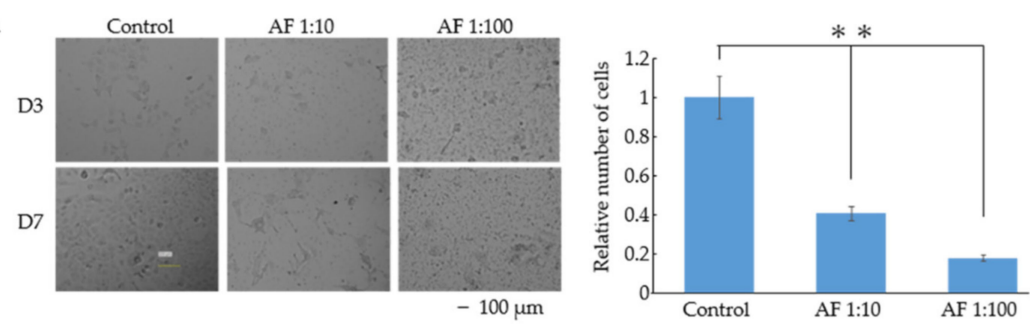

B
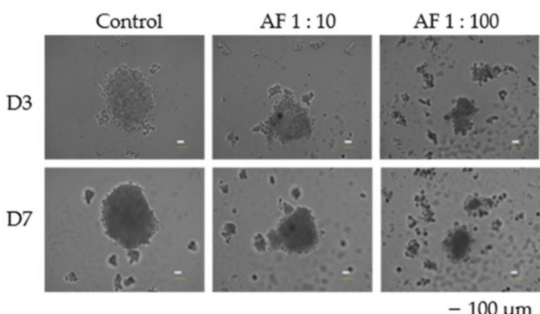

$-100 \mu \mathrm{m}$
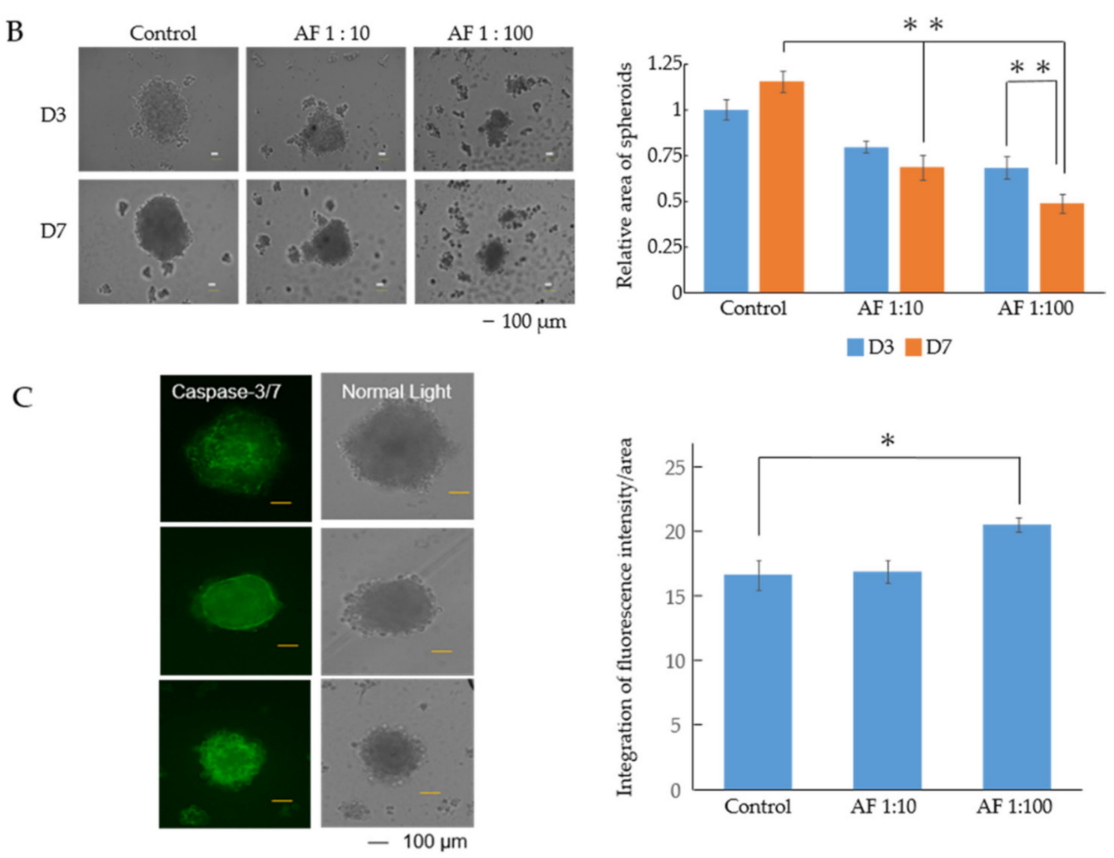

Figure 1. Heat-inactivated AF293 conidia suppress the growth of A549 cells. (A) Morphological changes and numbers of A549 cells with AF293 conidia at ratios of 1:10 and 1:100 on days 3 and 7 in 2D culture. AF 1:10 and AF 1:100 represents the number of AF293 conidia are 10- and 100-fold higher than that of A549 cells. D3: day 3, D7: day 7, Scale bar: $100 \mu \mathrm{m}$. Right panel represents the relative number of cells. The mean \pm standard deviation (SD) from three technical replicates is shown. ${ }^{* *} p<0.001$. (B) Morphological changes and relative area of A549 spheroids with AF293 conidia at ratios of 1:10 and 1:100 on days 3 and 7 in 3D culture. The right panel represents the area of spheroids. The mean \pm SD from three technical replicates is shown. ${ }^{* *} p<0.001$. (C) Representative signals for caspase-3/7 in A549 spheroids from three technical replicates (scale bar: $100 \mu \mathrm{m})$. The right panel represents the integration of fluorescence intensity/area. The mean \pm SD from three technical replicates is shown. ${ }^{*} p<0.01$. All data are representative of three independent experiments. 
In 3D culture, control A549 cells without AF293 conidia formed spheroids. However, A549 cells with AF293 conidia (1:10 and 1:100) formed incomplete spheroids, a trend that was more evident in the 1:100 group than in the 1:10 group (Figure 1B). The relative areas of A549 spheroids with AF293 conidia at ratios of 1:10 and 1:100 was 0.59- and 0.42-fold smaller than those of the control at day 7 , respectively (Figure 1B; $* * 0<0.001$ ). The relative area of A549 spheroids with AF293 conidia at a ratio of 1:100 on day 7 was 0.716 -fold smaller than that on day 3 (Figure 1B; ${ }^{* *} p<0.001$ ). These results suggest that AF293 conidia suppress the growth of epithelial cells.

To detect the involvement of apoptosis in the growth suppression, the apoptotic reaction of caspase- $3 / 7$ was observed in spheroids with AF conidia. The signal in the spheroid with AF293 conidia challenge at a ratio of 1:10 was not significantly different; however, the signal at a ratio of 1:100 was 1.23-fold larger than that of control, suggesting that AF293 conidia induce luminal apoptosis (Figure $1 C ;{ }^{*} p<0.01$ ). These results suggest the involvement of apoptosis in the efficacy of growth suppression by AF293 conidia.

\subsection{An RNA-Seq Analysis of Genes Induced by AF293 Conidia in A549 Cells}

To explore the effect of AF293 conidia on A549 cells, RNA-seq was performed. We selected genes showing a 2-fold increase of mRNA expression at a ratio of 1:10 compared with the control by AF293 conidia challenge (Supplementary Table S1). We found that 10 genes showed a 2-fold increase in mRNA expression at a ratio of 1:100 compared with that of 1:10 by the AF293 conidia challenge (Table 1). An ontology analysis of these genes revealed that the expression of genes associated with interferon (IFN) signaling, including MX1, OASL, RSAD2 and TRIM22, were increased by AF293 conidia (Table 2). Real-time quantitative reverse transcription-polymerase chain reaction (qRT-PCR) was performed to confirm the mRNA expression of these genes (Figure 2). As shown in the RNA-seq analyses (Table 1), the mRNA expression of these genes was significantly increased at a ratio of 1:10 in comparison to control by an AF293 conidia challenge (Figure $2 \mathrm{~A} ;{ }^{*} p<0.01$; $\left.{ }^{* *} p<0.001\right)$, and was further increased at a ratio of 1:100 in comparison to the ratio of 1:10 by the AF293 conidia challenge (Figure 2B). The increased expression of MX1 is dominant in comparison to that of OASL $(* p<0.01), \operatorname{RSAD2}\left({ }^{*} p<0.01\right)$, and TRIM22 $\left({ }^{* *} p<0.001\right)$, suggesting the critical roles of MX1 during AF293 conidia challenge. Notably, ontology analyses revealed that the downregulated genes at a ratio of 1:100 in comparison to the ratio of 1:10 by the AF293 conidia challenge was associated with the cell cycle among genes for which the expression at a ratio of 1:10 was not changed from that of the control (Figure 3, Supplementary Table S2), suggesting an association with the induction of apoptosis by AF conidia in A549 cells.

Table 1. Genes upregulated by AF293 conidia.

\begin{tabular}{cccc}
\hline Gene Symbol & $\mathbf{1 : 1 0 / C o n t r o l}$ & $\mathbf{1 : 1 0 0 / C o n t r o l}$ & $\mathbf{1 : 1 0 0 / 1 : 1 0}$ \\
\hline MX1 & 2.53020134 & 189.2080537 & 74.77984085 \\
RSAD2 & 2.48148148 & 75.2962963 & 30.34328358 \\
OASL & 2.88034188 & 50.98290598 & 17.70029674 \\
CMPK2 & 2.19444444 & 33.41666667 & 15.2278481 \\
PARP10 & 2.03125 & 19.90625 & 9.8 \\
TRIM22 & 2.81481481 & 16.65432099 & 5.916666667 \\
RARRES3 & 3.39285714 & 14.46428571 & 4.263157895 \\
SAMD9L & 2.60608622 & 10.30092984 & 3.952643529 \\
SSPO & 2.075 & 7.4625 & 3.596385542 \\
KNDC1 & 2.56603774 & 6.132075472 & 2.389705882 \\
GOLGA8EP & 2.11111111 & 4.555555556 & 2.157894737 \\
AC068533.7 & 3.34883721 & 6.953488372 & 2.076388889 \\
\hline
\end{tabular}


Table 2. Ontology analysis results.

\begin{tabular}{ccccc}
\hline Name of Pathway & Set Size & Candidates Contained & $p$-Value & Genes \\
\hline Interferon signaling & 158 & $4(2.5 \%)$ & $<0.0001$ & MX1, OASL, TRIM22, RSAD2 \\
Interferon alpha/beta signaling & 70 & $3(4.3 \%)$ & $<0.0001$ & MX1, OASL, RSAD2 \\
Cytokine signaling in immune system & 458 & $4(0.9 \%)$ & $<0.0001$ & MX1, OASL, TRIM22, RSAD2 \\
Interferon gamma signaling & 94 & $2(2.1 \%)$ & 0.001 & OASL, TRIM22 \\
Immune system & 1840 & $4(0.2 \%)$ & 0.001 & MX1, OASL, TRIM22, RSAD2 \\
\hline
\end{tabular}

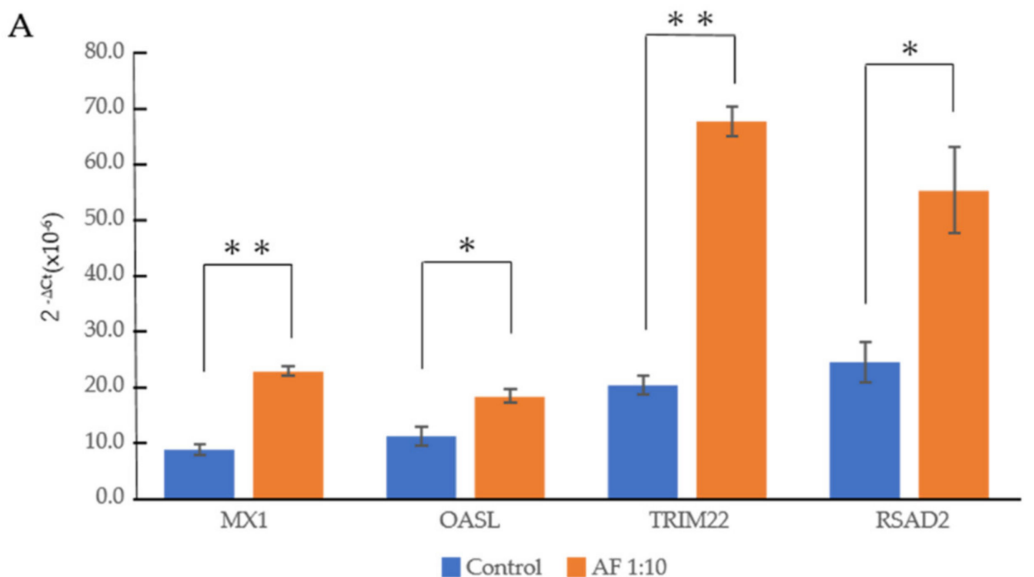

B

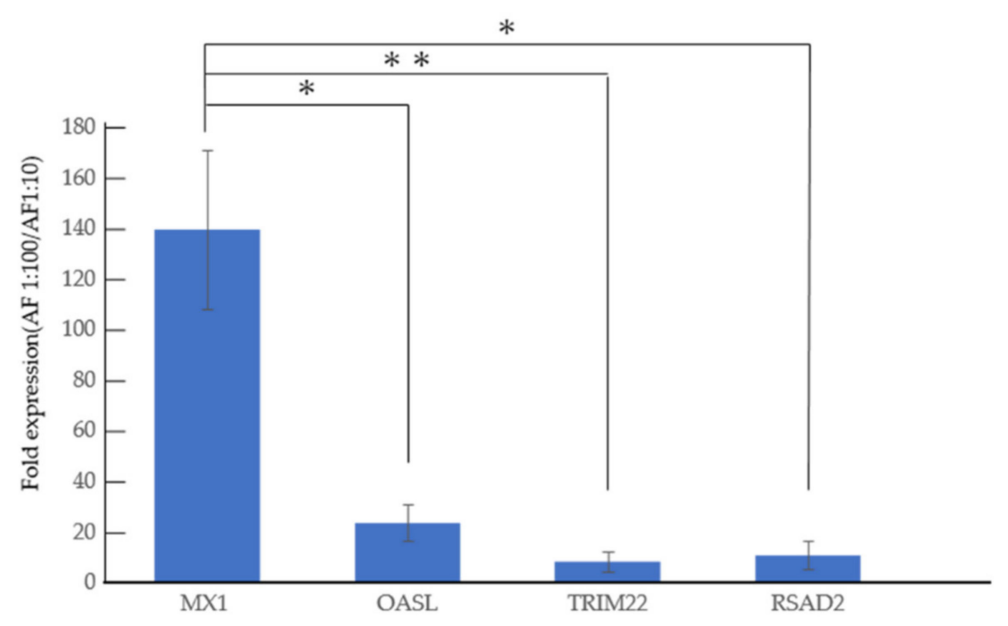

Figure 2. Heat-inactivated AF293 conidia increase the mRNA expression of MX1, OASL, TRIM22 and RSAD2 in A549 cells. (A) The mRNA expression of MX1, OASL, TRIM22 and RSAD2 in A549 cells with AF293 conidia at a ratio of 1:10 on day 3 in $2 \mathrm{D}$ culture. The $X$ axis represents the expression value $\left[2^{-\Delta \mathrm{Ct}}\left(\times 10^{-6}\right)\right]$. (B) The relative mRNA expression of MX1, OASL, TRIM22 and RSAD2 in A549 cells with AF293 conidia at ratios of 1:100 in comparison to those at a ratio of 1:10 on day 3 in $2 \mathrm{D}$ culture. The mean \pm SD from three biological replicates is shown. ${ }^{*} p<0.01,{ }^{* *} p<0.001$. 

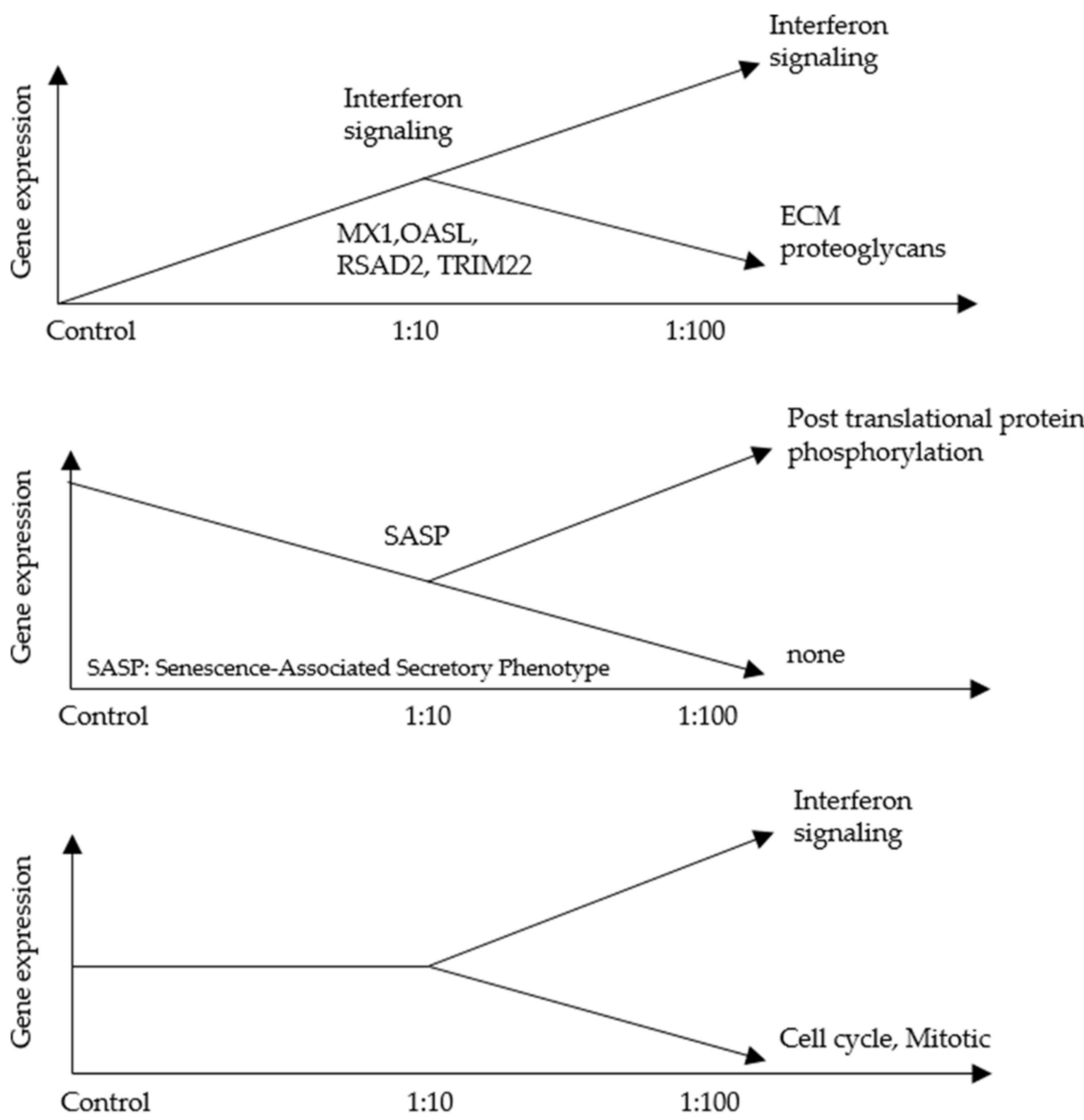

Figure 3. A schematic representation of the relative mRNA expression, ontology and related genes from RNA-seq data of A549 cells with AF293 conidia at ratios of 1:10 and 1:100 on day 3 in 2D culture. The $X$ axis represents the gene expression levels.

\subsection{AF293 Conidia Did Not Increase the Expression of Epithelial-Mesenchymal Transition} (EMT)-Related Genes in A549 Cells

To investigate whether or not heat-inactivated AF conidia causes EMT in A549, we cultured A549 with AF293 conidia at a ratio of 1:100 in 2D culture. The expression of ZO-1 (an epithelial marker) showed a 0.89-fold decrease, while that of e-cadherin (another epithelial marker) was increased 1.32-fold, suggesting that the change in epithelial markers is inconsistent. There was no significant change in the expression of EMT-associated proteins, vimentin (Figure 4), suggesting that AF293 conidia did not affect the expression of EMT-related genes in A549 cells. 


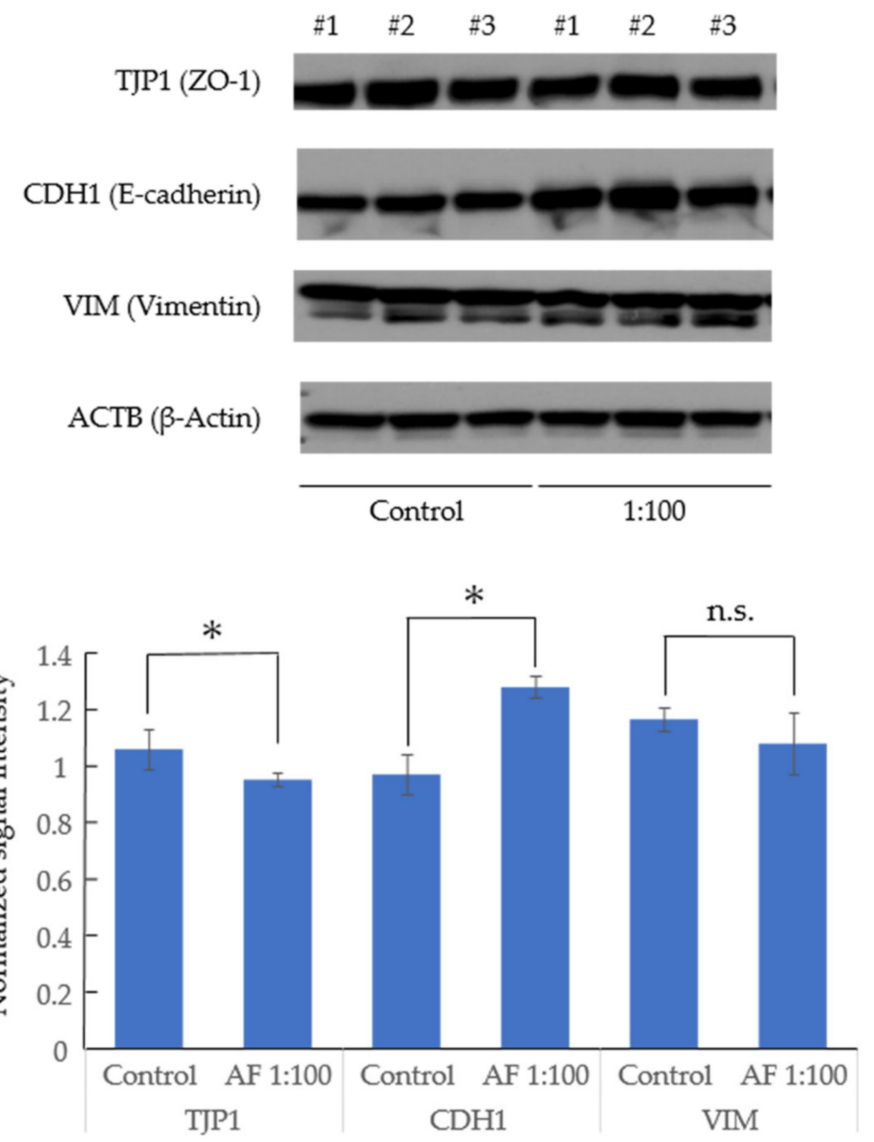

Figure 4. The assessment of the expression of epithelial-mesenchymal transition (EMT)-related markers in A549 cells by Western blotting. The expression of the indicated proteins in the lysates was examined using specific antibodies for TJP1 (ZO-1), CDH1 (e-cadherin), VIM (vimentin) and ACTB ( $\beta$-actin). Actin was used as a loading control. The left-half columns (\#1-3) show the findings for the A549 cells in the control group. The right-half columns (\#1-3) show the findings for the A549 cells with AF293 conidia at a ratio of 1:100. AF293 conidia did not increase the expression of EMT-related genes in A549 cells. Data are representative of three independent experiments. The lower panel represents the signal intensity normalized by the intensity of ACTB. The mean \pm SD from three biological replicates is shown. ${ }^{*} p<0.05$. n.s., not significant.

\subsection{AF293 Conidia Promoted the Growth of Mouse NIH/3T3 Cells}

To compare the effects of AF293 on A549 cells, we examined the effects of AF293 on mouse NIH/3T3 cells. 3T3 cells were cultured with heat-inactivated AF293 conidia in the same way. In 2D culture, the growth of 3T3 cells with or without heat-inactivated AF293 (1:10 and 1:100) did not differ to a statistically significant extent (Figure 5A).

In 3D culture, control 3T3 cells formed spheroids; 3T3 cells with AF293 (1:100) formed even larger spheroids than those in the control group. (Figure 5B). The relative areas of 3T3 spheroids with AF293 conidia at 1:100 was 2.41- and 2.38-fold larger than those of the control and those at 1:10 on day 7, respectively (Figure $5 \mathrm{~B} ;{ }^{* *} p<0.01$ ). The relative area of 3T3 spheroids with AF293 conidia at a ratio of 1:100 on day 7 was 1.39-fold larger than that on day 3 (Figure $5 \mathrm{~B} ;{ }^{*} p<0.1$ ). These results suggest that AF293 conidia promoted the growth of 3T3 cells in a 3D-specific way. The signal of caspase-3/7 was not completely detected in any spheroids with AF293 conidia (Figure 5C). 
A

Control
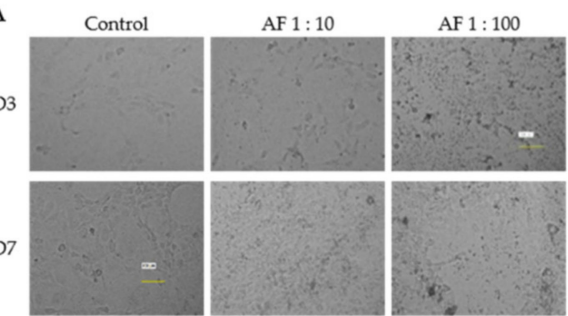

D7
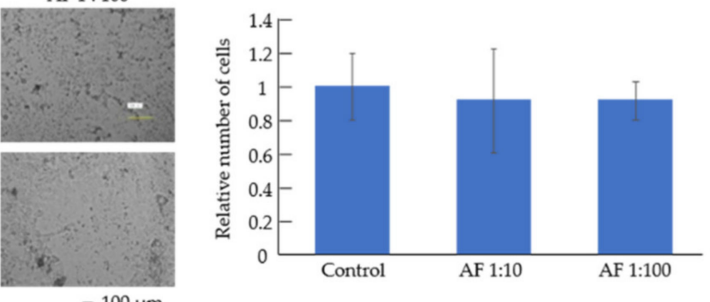

B
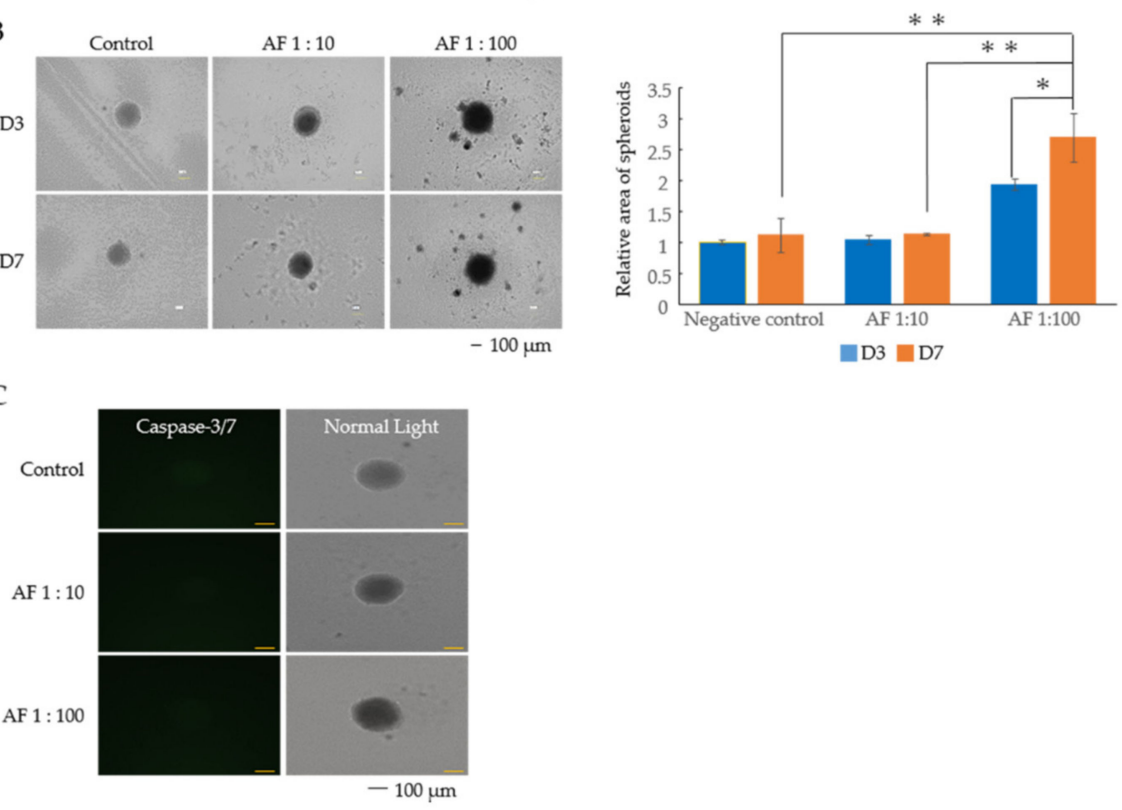

Figure 5. Heat-inactivated AF293 conidia promoted the growth of mouse NIH/3T3 cells. (A) Morphological changes in mouse NIH/3T3 cells with AF293 conidia at ratios of 1:10 and 1:100 on days 3 and 7 in 2D culture. AF 1:10 and AF 1:100 represents the number of AF293 conidia are 10and 100-fold higher than that of mouse NIH/3T3 cells. D3: day 3, D7: day 7, scale bar: $100 \mu \mathrm{m}$. Right panel represents the relative number of cells. The mean \pm SD from three technical replicates is shown. (B) Morphological changes and the relative area of mouse NIH/3T3 cells spheroids with AF293 conidia at ratios of 1:10 and 1:100 on day 7 in 3D culture. Right panel represents the area of spheroids. The mean \pm SD from three technical replicates is shown. ${ }^{*} p<0.001,{ }^{* *} p<0.01$. (C) Representative signals for caspase-3/7 in mouse NIH/3T3 spheroids from three technical replicates (Scale bar: $100 \mu \mathrm{m}$ ). All data are representative of three independent experiments.

\subsection{An RNA-Seq Analysis of Genes Induced by AF293 Conidia in NIH/3T3 Cells}

To explore the effect of AF293 conidia on NIH/3T3 cells, RNA-seq was performed. We selected genes for which the mRNA expression showed a 2-fold increase at a ratio of 1:10 in comparison to control in response to an AF293 conidia challenge (Supplementary Table S3). We found that 25 genes showed for which the mRNA expression showed a 2-fold increase at a ratio of 1:100 in comparison to the expression at 1:10 in response to an AF293 conidia challenge (Table 3). An ontology analysis of these genes revealed that the expression of genes associated with IFN signaling, including Oas2, Mx2 and Rsad2, was increased by AF293 conidia (Table 4). Notably, the ontology analyses revealed that, among the genes for which the expression did not change between a ratio of 1:10 and control, the genes that were downregulated at a ratio of 1:100 in comparison to a ratio of 1:10 in response to an AF293 conidia challenge were not associated with the cell cycle (Figure 6), suggesting that AF conidia does not induce apoptosis in NIH/3T3 cells. 
Table 3. Upregulated genes by Af293 conidia.

\begin{tabular}{|c|c|c|c|c|c|c|}
\hline Gene Symbol & 1:10/Control & $p$-Value & 1:100/Control & $p$-Value & 1:100/1:10 & $p$-Value \\
\hline Oas2 & 17.6 & 0.01418642 & 227.0666667 & 0.000142248 & 12.90151515 & 0.000217291 \\
\hline Oasl2 & 12.8181818 & 0.00105008 & 153.6439394 & $2.98985 \times 10^{-8}$ & 11.98640662 & $1.97743 \times 10^{-7}$ \\
\hline Oas1a & 3.90163934 & 0.00115744 & 44.29508197 & $4.56841 \times 10^{-5}$ & 11.35294118 & $6.24434 \times 10^{-5}$ \\
\hline Mx2 & 9.27692308 & 0.00694089 & 103.4923077 & $1.36501 \times 10^{-6}$ & 11.15588723 & $4.53558 \times 10^{-6}$ \\
\hline Oas1g & 3.666666667 & 0.00878991 & 35.48888889 & $8.18609 \times 10^{-5}$ & 9.678787879 & 0.000126154 \\
\hline Usp18 & 2.55406912 & 0.00924884 & 20.79487179 & $9.33815 \times 10^{-5}$ & 8.14185945 & 0.000131795 \\
\hline Zbp1 & 2.7 & 0.08168567 & 21.74 & 0.000237643 & 8.051851852 & 0.000479164 \\
\hline Isg15 & 4.02118003 & 0.01702177 & 30.3494705 & $9.91865 \times 10^{-5}$ & 7.547404063 & 0.000208583 \\
\hline Bst2 & 3.45379024 & 0.00064043 & 25.64382139 & $3.26371 \times 10^{-6}$ & 7.424834636 & $6.17672 \times 10^{-6}$ \\
\hline Rtp4 & 3.24145786 & 0.01492393 & 21.29840547 & $9.5736 \times 10^{-5}$ & 6.570625439 & 0.000206501 \\
\hline Gbp3 & 4.82394366 & 0.004038 & 29.6971831 & 0.000166256 & 6.15620438 & 0.000345417 \\
\hline $\operatorname{Irgm} 2$ & 7.52132701 & 0.005725 & 45.36966825 & $4.0298 \times 10^{-5}$ & 6.032136106 & 0.000125601 \\
\hline Rsad2 & 2.56321839 & 0.00226457 & 14.55172414 & $5.23578 \times 10^{-5}$ & 5.677130045 & $8.98531 \times 10^{-5}$ \\
\hline Apol9a & 2.4 & 0.00182007 & 12.2691358 & 0.000515447 & 5.112139918 & 0.000880427 \\
\hline Ifit3 & 3.18018018 & 0.01196764 & 16.25675676 & $3.98466 \times 10^{-6}$ & 5.111898017 & $3.48212 \times 10^{-5}$ \\
\hline Xaf1 & 6.21891419 & 0.00600125 & 30.08231173 & $5.8742 \times 10^{-6}$ & 4.83722895 & $6.10851 \times 10^{-5}$ \\
\hline Irf7 & 2.27311828 & 0.00105694 & 10.51397849 & 0.000500941 & 4.625354778 & 0.000872438 \\
\hline Oas1b & 4.12820513 & 0.02932599 & 18.07692308 & $7.73289 \times 10^{-5}$ & 4.378881988 & 0.000548655 \\
\hline Ifit1 & 4.65353038 & 0.00588437 & 18.42857143 & $2.18323 \times 10^{-7}$ & 3.960127029 & $4.76394 \times 10^{-5}$ \\
\hline Lgals3bp & 2.61022273 & 0.00030663 & 9.311536265 & 0.000139853 & 3.56733399 & 0.000351664 \\
\hline Helz2 & 2.16917969 & $7.7487 \times 10^{-5}$ & 6.965140407 & $8.40955 \times 10^{-7}$ & 3.210955934 & $2.64734 \times 10^{-6}$ \\
\hline Ddx58 & 2.93550577 & 0.00063444 & 9.393075356 & $1.45365 \times 10^{-5}$ & 3.199814986 & $7.08851 \times 10^{-5}$ \\
\hline Parp9 & 2.15489614 & 0.00044662 & 5.903264095 & $7.46111 \times 10^{-6}$ & 2.739465712 & $2.5749 \times 10^{-5}$ \\
\hline Irf9 & 2.77596342 & 0.00099975 & 7.331809275 & $2.31458 \times 10^{-5}$ & 2.641176471 & 0.000182173 \\
\hline Apol8 & 2.20883534 & 0.00066116 & 4.977911647 & 0.000122632 & 2.253636364 & 0.000723058 \\
\hline
\end{tabular}

Table 4. Ontology analysis of genes shown in Table 3.

\begin{tabular}{|c|c|c|c|}
\hline Name of Pathway & Set Size & Candidates Contained & $p$-Value \\
\hline Interferon Signaling & 158 & $13(8.2 \%)$ & $1.76 \times 10^{-22}$ \\
\hline Interferon alpha/beta signaling & 70 & $11(15.7 \%)$ & $5.88 \times 10^{-22}$ \\
\hline Cytokine Signaling in Immune system & 458 & $13(2.8 \%)$ & $2.30 \times 10^{-16}$ \\
\hline Immune System & 1840 & $14(0.8 \%)$ & $5.06 \times 10^{-10}$ \\
\hline ISG15 antiviral mechanism & 30 & $4(13.3 \%)$ & $5.38 \times 10^{-8}$ \\
\hline Antiviral mechanism by IFN-stimulated genes & 30 & $4(13.3 \%)$ & $5.38 \times 10^{-8}$ \\
\hline Interferon gamma signaling & 94 & $4(4.3 \%)$ & $5.69 \times 10^{-6}$ \\
\hline DDX58/IFIH1-mediated induction of interferon-alpha/beta & 56 & $3(5.4 \%)$ & $4.94 \times 10^{-5}$ \\
\hline TRAF3-dependent IRF activation pathway & 13 & $2(15.4 \%)$ & 0.000125 \\
\hline TRAF6 mediated IRF7 activation & 17 & $2(11.8 \%)$ & 0.000217 \\
\hline Negative regulators of DDX58/IFIH1 signaling & 23 & $2(8.7 \%)$ & 0.000401 \\
\hline Cytosolic sensors of pathogen-associated DNA & 51 & $2(3.9 \%)$ & 0.00198 \\
\hline Innate Immune System & 1077 & $5(0.5 \%)$ & 0.00985 \\
\hline
\end{tabular}



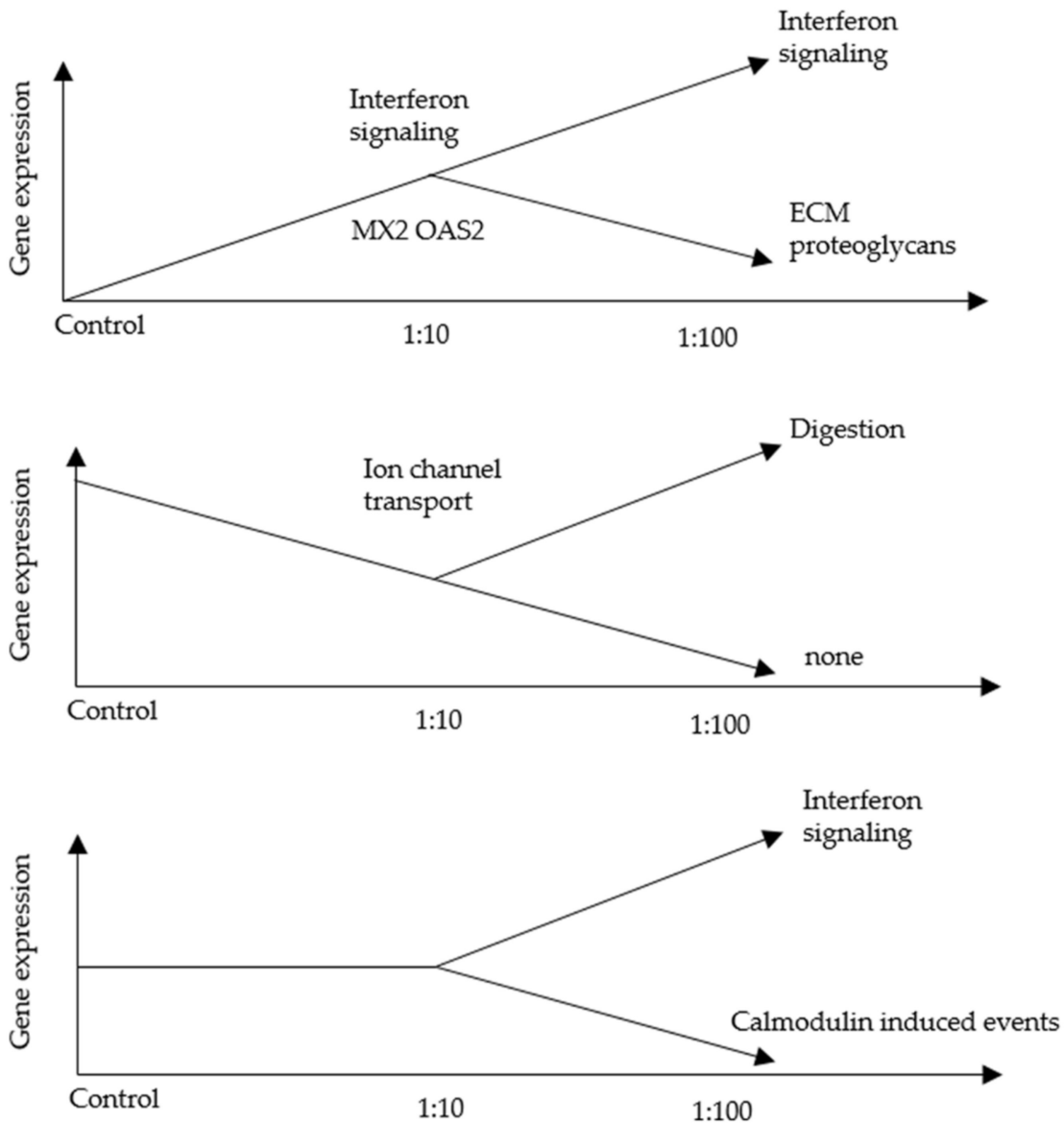

Figure 6. A schematic representation of the relative mRNA expression, ontology and related genes from RNA-seq data of NIH/3T3 cells with AF293 conidia at ratios of 1:10 and 1:100 on day 3 in 2D culture. The $\mathrm{X}$ axis represents the of gene expression levels.

\section{Discussion}

In the present study, we performed 2D and 3D culture with heat-inactivated AF293 conidia using human lung epithelial A549 cells and found that AF293 conidia reduced the proliferation of A549 cells in both 2D and 3D cultures. Furthermore, we performed RNA-seq using 2D-cultured A549 cells with AF293 conidia and detected early response genes associated with IFN signaling, such as MX1, OASL, RSAD2 and TRIM22. Notably, type-I IFN acts on cells during viral infections to inhibit viral replication [22]. Indeed, a recent study revealed the expression of these genes in A549 cells infected by influenza virus [23].

An ontology analysis failed to show any apparent association between AF293 conidia and apoptosis; however, cell cycle genes were downregulated by high concentrations of AF293 conidia; Figure 3, Supplementary Table S2). This suggests that apoptosis was induced by a checkpoint mechanism. Furthermore, EMT was not clearly observed (Figure 4), suggesting that a longer exposure or higher concentration administration may be needed for EMT induction.

It was recently hypothesized that idiopathic pulmonary fibrosis might be a neoproliferative disorder of the lung because this disease exhibits several pathogenic features similar to cancer [24]. Surprisingly, our study showed that AF conidia increase the growth of NIH/3T3 spheroids despite no significant change in 2D culture (Figure 5). Ontology analyses of RNA-seq data shows similar patterns with those seen in A549 cells. For example, genes associated with IFN signaling, such as Oas2, Mx2 and Rsad2, are upregulated by dose dependent manner. However, downregulation of genes associated with cell cycle 
is not observed. Humans possess two MX genes, MX1 and MX2, with a high level of homology [25]. MX protein is considered as a key mediator of the IFN-induced antiviral response. MX proteins contain the typical GTP-binding motif and show significant homology to the dynamin family of GTPases. Strong interaction of MX proteins with tubulin suggests that Mx proteins could be involved in mitosis [26,27]. However, roles of MX proteins in proliferation and apoptosis are inconsistent. Indeed, the increased expression of MX1 is associated with liver fibrosis and high MX1 protein expression is associated with increased proliferation and poor prognosis in breast cancer [28,29]. On the other hand, the gain of MX1 expression in in human prostate cancer $\mathrm{LNCaP}$ cells resulted in cell cycle arrest [26]. Regarding MX2 protein, overexpression of MX2 in primary melanoma reduces in vivo proliferation partially through inhibition of AKT activation. However, in a subset of melanoma cell lines with high endogenous MX2 expression where downregulation of MX2 leads to reduced proliferation [30]. Our results also demonstrate that AF conidia promote $3 \mathrm{~T} 3$ proliferation in 3DF but not in 2D. The structure and distribution of the cytoskeleton is profoundly influenced by the dimensionality of a cell's environment [31]. These results suggest that the binding between MX proteins and cytoskeleton is influenced by 2D or 3D culture and cellular response by AF conidia is context-dependent possibly through critical roles of MX proteins for the cytoskeleton. Interestingly, the increased expression of MX1 autoantibody in serum is associated with a good prognosis in patients with idiopathic interstitial pneumonia [32], suggesting that the inhibition of MX proteins is a possible strategy for preventing fibrotic lung diseases.

In conclusion, our study showed that high concentrations of AF293 conidia induce A549 apoptosis along with IFN inducible genes, such as MX1. In addition, AF293 conidia increase NIH/3T3 proliferation possibly through IFN signaling, such as MX2 induction. These results suggest the role of MX proteins during colonization. Although several mechanisms for epithelial apoptosis and fibroblastic proliferation were postulated [33], whether or not different responses of these two types of cell to AF293 conidia are involved in the development of pulmonary fibrosis remains unclear. More detailed analyses are expected to help clarify the spatiotemporal regulation of AF293 conidia, resulting in further understanding of the pathogenesis of Aspergillus spp. in pulmonary fibrosis.

Supplementary Materials: The following are available online at https:/ /www.mdpi.com/article/10 $.3390 /$ microorganisms $9071435 /$ s1.

Author Contributions: H.K., T.M. and T.T. performed experiments, analyzed the data and wrote a manuscript draft. K.I. and Y.K. participated in the study design, data collection and analysis. S.S., M.F. and H.I. conceived the idea and designed the study, interpreted the data, provided important intellectual content, and obtained the final approval of the submitted manuscript. All authors have read and agreed to the published version of the manuscript.

Funding: This work was supported by a Grant-in-Aid for Scientific Research (C) (KAKENHI, Grant Number 18K15154) from the Ministry of Education, Culture, Sports, Science.

Institutional Review Board Statement: Not applicable.

Informed Consent Statement: Not applicable.

Data Availability Statement: Not applicable.

Acknowledgments: We thank Tashiro, M. and Takazono, T. (Nagasaki University) for their kind and generous gift of the strain of Aspergillus fumigatus. We also thank Shiroyama, N. for her technical assistance.

Conflicts of Interest: The authors declare no competing interests.

\section{References}

1. Sabino, R.; Veríssimo, C.; Viegas, C.; Viegas, S.; Brandão, J.; Alves-Correia, M.; Borrego, L.M.; Clemons, K.V.; Stevens, D.A.; Richardson, M. The role of occupational aspergillus exposure in the development of diseases. Med. Mycol. 2019, 57 (Suppl. 2), S196-S205. [CrossRef] 
2. Tashiro, T.; Izumikawa, K.; Tashiro, M.; Takazono, T.; Morinaga, Y.; Yamamoto, K.; Imamura, Y.; Miyazaki, T.; Seki, M.; Kakeya, H.; et al. Diagnostic significance of Aspergillus species isolated from respiratory samples in an adult pneumology ward. Med. Mycol. 2011, 49, 581-587. [CrossRef] [PubMed]

3. Walsh, T.J.; Anaissie, E.J.; Denning, D.W.; Herbrecht, R.; Kontoyiannis, D.P.; Marr, K.A.; Morrison, V.A.; Segal, B.H.; Steinbach, W.J.; Stevens, D.A.; et al. Treatment of aspergillosis: Clinical practice guidelines of the Infectious Diseases Society of America. Clin. Infect Dis. 2008, 46, 327-360. [CrossRef] [PubMed]

4. Raghu, G.; Chen, S.Y.; Yeh, W.S.; Maroni, B.; Li, Q.; Lee, Y.C.; Collard, H.R. Idiopathic pulmonary fibrosis in US Medicare beneficiaries aged 65 years and older: Incidence, prevalence, and survival, 2001-2011. Lancet Respir. Med. 2014, 2, 566-572. [CrossRef]

5. Strongman, H.; Kausar, I.; Maher, T.M. Incidence, Prevalence, and Survival of Patients with Idiopathic Pulmonary Fibrosis in the UK. Adv. Ther. 2018, 35, 724-736. [CrossRef]

6. Yokohori, N.; Aoshiba, K.; Nagai, A.; Respiratory Failure Research Group in Japan. Increased levels of cell death and proliferation in alveolar wall cells in patients with pulmonary emphysema. Chest 2004, 125, 626-632. [CrossRef] [PubMed]

7. Kuwano, K.; Kunitake, R.; Kawasaki, M.; Nomoto, Y.; Hagimoto, N.; Nakanishi, Y.; Hara, N. P21Waf1/Cip1/Sdi1 and p53 expression in association with DNA strand breaks in idiopathic pulmonary fibrosis. Am. J. Respir Crit. Care Med. 1996, 154, 477-483. [CrossRef]

8. Cottin, V.; Nunes, H.; Brillet, P.Y.; Delaval, P.; Devouassoux, G.; Tillie-Leblond, I.; Israel-Biet, D.; Court-Fortune, I.; Valeyre, D.; Cordier, J.F.; et al. Combined pulmonary fibrosis and emphysema: A distinct underrecognised entity. Eur. Respir J. 2005, 26, 586-593. [CrossRef] [PubMed]

9. Tanaka, T.; Yoshimi, M.; Maeyama, T.; Hagimoto, N.; Kuwano, K.; Hara, N. Resistance to Fas-mediated apoptosis in human lung fibroblast. Eur. Respir J. 2002, 20, 359-368. [CrossRef]

10. Kuwano, K.; Hagimoto, N.; Nakanishi, Y. The role of apoptosis in pulmonary fibrosis. Histol. Histopathol. 2004, 19, 867-881. [CrossRef]

11. Yamada, M.; Kuwano, K.; Maeyama, T.; Hamada, N.; Yoshimi, M.; Nakanishi, Y.; Kasper, M. Dual-immunohistochemistry provides little evidence for epithelial-mesenchymal transition in pulmonary fibrosis. Histochem. Cell Biol. 2008, 129, 453-462. [CrossRef] [PubMed]

12. Fukuda, Y.; Homma, T.; Suzuki, S.; Takuma, T.; Tanaka, A.; Yokoe, T.; Ohnishi, T.; Niki, Y.; Sagara, H. High burden of Aspergillus fumigatus infection among chronic respiratory diseases. Chron. Respir Dis. 2018, 15, 279-285. [CrossRef] [PubMed]

13. Beisswenger, C.; Hess, C.; Bals, R. Aspergillus fumigatus conidia induce interferon- $\beta$ signalling in respiratory epithelial cells. Eur. Respir J. 2012, 39, 411-418. [CrossRef]

14. Berkova, N.; Lair-Fulleringer, S.; Féménia, F.; Huet, D.; Wagner, M.C.; Gorna, K.; Tournier, F.; Ibrahim-Granet, O.; Guillot, J.; Chermette, R.; et al. Aspergillus fumigatus conidia inhibit tumour necrosis factor- or staurosporine-induced apoptosis in epithelial cells. Int. Immunol. 2006, 18, 139-150. [CrossRef]

15. Tsunoda, T.; Takashima, Y.; Fujimoto, T.; Koyanagi, M.; Yoshida, Y.; Doi, K.; Tanaka, Y.; Kuroki, M.; Sasazuki, T.; Shirasawa, S. Three-dimensionally specific inhibition of DNA repair-related genes by activated KRAS in colon crypt model. Neoplasia 2010, 12, 397-404. [CrossRef] [PubMed]

16. Foster, K.A.; Oster, C.G.; Mayer, M.M.; Avery, M.L.; Audus, K.L. Characterization of the A549 cell line as a type II pulmonary epithelial cell model for drug metabolism. Exp. Cell Res. 1998, 243, 359-366. [CrossRef]

17. Blasi, E.; Brozzetti, A.; Francisci, D.; Neglia, R.; Cardinali, G.; Bistoni, F.; Vidotto, V.; Baldelli, F. Evidence of microevolution in a clinical case of recurrent Cryptococcus neoformans meningoencephalitis. Eur. J. Clin. Microbiol. Infect Dis. 2001, 20, 535-543. [CrossRef]

18. Tsunoda, T.; Ishikura, S.; Doi, K.; Iwaihara, Y.; Hidesima, H.; Luo, H.; Hirose, Y.; Shirasawa, S. Establishment of a Threedimensional Floating Cell Culture System for Screening Drugs Targeting KRAS-mediated Signaling Molecules. Anticancer Res. 2015, 35, 4453-4459.

19. Nishi, K.; Luo, H.; Ishikura, S.; Doi, K.; Iwaihara, Y.; Wills, L.; Baillie, G.S.; Sakata, T.; Shirasawa, S.; Tsunoda, T. Apremilast Induces Apoptosis of Human Colorectal Cancer Cells with Mutant KRAS. Anticancer Res. 2017, 37, 3833-3839. [CrossRef]

20. Tsunoda, T.; Ota, T.; Fujimoto, T.; Doi, K.; Tanaka, Y.; Yoshida, Y.; Ogawa, M.; Matsuzaki, H.; Hamabashiri, M.; Tyson, D.R.; et al. Inhibition of phosphodiesterase-4 (PDE4) activity triggers luminal apoptosis and AKT dephosphorylation in a 3-D colonic-crypt model. Mol. Cancer 2012, 11, 46. [CrossRef]

21. Kamburov, A.; Stelzl, U.; Lehrach, H.; Herwig, R. The ConsensusPathDB interaction database: 2013 update. Nucleic Acids Res. 2013, 41, D793-D800. [CrossRef]

22. Hall, J.C.; Rosen, A. Type I interferons: Crucial participants in disease amplification in autoimmunity. Nat. Rev. Rheumatol. 2010, 6, 40-49. [CrossRef]

23. Jiao, P.; Fan, W.; Cao, Y.; Zhang, H.; Tian, L.; Sun, L.; Luo, T.; Liu, W.; Li, J. Robust induction of interferon and interferon-stimulated gene expression by influenza B/Yamagata lineage virus infection of A549 cells. PLoS ONE 2020, 15, e0231039. [CrossRef]

24. Vancheri, C. Idiopathic pulmonary fibrosis: An altered fibroblast proliferation linked to cancer biology. Proc. Am. Thorac. Soc. 2012, 9, 153-157. [CrossRef]

25. Haller, O.; Staeheli, P.; Schwemmle, M.; Kochs, G. Mx GTPases: Dynamin-like antiviral machines of innate immunity. Trends Microbiol. 2015, 23, 154-163. [CrossRef] 
26. Brown, S.G.; Knowell, A.E.; Hunt, A.; Patel, D.; Bhosle, S.; Chaudhary, J. Interferon inducible antiviral MxA is inversely associated with prostate cancer and regulates cell cycle, invasion and Docetaxel induced apoptosis. Prostate 2015, 75, 266-279. [CrossRef] [PubMed]

27. Horisberger, M.A. Interferon-induced human protein MxA is a GTPase which binds transiently to cellular proteins. J. Virol. 1992, 66, 4705-4709. [CrossRef]

28. Domagalski, K.; Pawłowska, M.; Kozielewicz, D.; Dybowska, D.; Tretyn, A.; Halota, W. The Impact of IL28B Genotype and Liver Fibrosis on the Hepatic Expression of IP10, IFI27, ISG15, and MX1 and Their Association with Treatment Outcomes in Patients with Chronic Hepatitis C. PLoS ONE 2015, 10, e0130899. [CrossRef] [PubMed]

29. Aljohani, A.I.; Joseph, C.; Kurozumi, S.; Mohammed, O.J.; Miligy, I.M.; Green, A.R.; Rakha, E.A. Myxovirus resistance 1 (MX1) is an independent predictor of poor outcome in invasive breast cancer. Breast Cancer Res. Treat 2020, 181, 541-551. [CrossRef] [PubMed]

30. Juraleviciute, M.; Pozniak, J.; Nsengimana, J.; Harland, M.; Randerson-Moor, J.; Wernhoff, P.; Bassarova, A.; Øy, G.F.; Trøen, G.; Flørenes, V.A.; et al. MX 2 is a novel regulator of cell cycle in melanoma cells. Pigment Cell Melanoma Res. 2020, 33, 446-457. [CrossRef]

31. Walker, M.; Rizzuto, P.; Godin, M.; Pelling, A.E. Structural and mechanical remodeling of the cytoskeleton maintains tensional homeostasis in 3D microtissues under acute dynamic stretch. Sci. Rep. 2020, 10, 7696. [CrossRef] [PubMed]

32. Hamano, Y.; Kida, H.; Ihara, S.; Murakami, A.; Yanagawa, M.; Ueda, K.; Honda, O.; Tripathi, L.P.; Arai, T.; Hirose, M.; et al. Classification of idiopathic interstitial pneumonias using anti-myxovirus resistance-protein 1 autoantibody. Sci. Rep. 2017, 7, 43201. [CrossRef] [PubMed]

33. Selman, M.; Pardo, A. Revealing the pathogenic and aging-related mechanisms of the enigmatic idiopathic pulmonary fibrosis. an integral model. Am. J. Respir Crit. Care Med. 2014, 189, 1161-1172. [CrossRef] [PubMed] 